\title{
Enhanced wild-type p53 expression by small activating RNA dsP53-285 induces cell cycle arrest and apoptosis in pheochromocytoma cell line PC12
}

\author{
DENGQIANG LIN ${ }^{1 *}$, LI MENG $^{1 *}$, FEIFEI XU ${ }^{1 *}$, JIANPO LIAN $^{1}$, YUNZE XU $^{2}$, \\ XIN XIE ${ }^{1}$, XIAOJING WANG ${ }^{1}$, HONGCHAO HE $^{1}, \mathrm{CHENGHE} \mathrm{WANG}^{1}$ and $\mathrm{YU} \mathrm{ZHU}^{1}$ \\ ${ }^{1}$ Department of Urology, Ruijin Hospital, Shanghai Jiaotong University, School of Medicine, \\ Shanghai 200025; ${ }^{2}$ Department of Urology, Renji Hospital Affiliated to the Medical \\ School of Shanghai Jiaotong University, Shanghai 200000, P.R. China
}

Received April 5, 2017; Accepted August 30, 2017

DOI: $10.3892 /$ or.2017.5993

\begin{abstract}
Malignant pheochromocytoma (PHEO) is diagnosed only when metastasis has occurred, making it less likely for patients to obtain the benefits of traditional chemotherapy. Anti-oncogene TP53 mutation has been detected in PHEO and is possibly related to disease progression. However, whether the upregulation of wild-type TP53 has antitumoral effects on PHEO remains completely unknown. In the present study, we used RNA activation (RNAa) technique to upregulate the expression of wild-type TP53 by transfecting synthetic dsP53-285 into PHEO cell line PC12. We found that the upregulation of wild-type p53 blocked the transition of PC12 cells from the G0/G1 to the S phase, with induction of apoptosis. Additionally, the above-mentioned findings were attested in vivo. Most importantly, dsP53-285-induced antitumoral effects were reversible following co-transfection with siRNA that targeted p53 mRNA. Collectively, our results revealed that the upregulation of p53 and possibly other anti-oncogenes may provide a potential effective therapeutic strategy for PHEO.
\end{abstract}

\section{Introduction}

Pheochromocytoma (PHEO) is a rare neuroendocrine tumor derived from the adrenal medulla and extra-adrenal neural crest chromaffin tissues, with an incidence of 2-8 cases/million/year. However, a higher than expected

Correspondence to: Dr Yu Zhu or Dr Chenghe Wang, Department of Urology, Ruijin Hospital, Shanghai Jiaotong University, School of Medicine, 197 Ruijin Er Road, Shanghai 200025, P.R. China

E-mail: zyyyhyq@126.com

E-mail: wangch8603@163.com

${ }^{*}$ Contributed equally

Key words: pheochromocytoma, small activating RNA, p53 incidence has been reported (1). Theoretically, radical resection remains the sole treatment option. Despite the relatively satisfactory outcome of an 84-96\% 5-year survival rate in patients with benign masses, the $<50 \%$ survival rate $(2-4)$ and up to $65.4 \%$ recurrence rate within 5 years (5) are the overwhelming statistics for patients with malignant PHEO. Apart from the gold standard of finding ectopic chromaffin tissue, there are no early diagnostic markers for PHEO and treatment is not optimal for patients with metastatic disease. In addition, alternative treatments are of little benefit to patients with metastatic disease. Therefore, there is an urgent need for new and effective therapeutic agents for PHEO patients, especially those with malignant tumors.

Cancer is generally considered a genomic disease that progresses by accumulating gene mutations leading to oncogene activation and/or anti-oncogene inactivation. TP53, as a common tumor-suppressor gene, is mutated in more than half of human cancers (6). Through the regulation of various target genes, the biological functions of p53 include DNA repair, cell cycle arrest and induction of apoptosis or senescence (7-9). Thus, inactivating TP53 mutations could result in tumorigenesis. By coincidence, numerous studies have revealed TP53 mutations in PHEO $(10,11)$. However, whether the 'artificial' upregulation of TP53 has anti-PHEO effects remains completely unknown.

In the present study, dsP53-285, a small double-stranded RNA (dsRNA) that targets the TP53 promoter was employed to induce wild-type TP53 expression in the PHEO cell line PC12. This phenomenon, termed RNA activation (RNAa), is conserved in mammalian species. The enhanced p53 expression caused cell cycle arrest and induced apoptosis in association with altered expression of the corresponding proteins and mRNAs, including cyclin D1, CDK4/6 and caspase 9 and 3. Finally, our results were further demonstrated using in vivo models.

\section{Materials and methods}

Cell line and main reagents. PHEO cell line (PC12) was originally donated by the Institute of Neurology in Ruijin 
Hospital Affiliated to the Medical School of Shanghai Jiaotong University. dsP53-285 was purchased from RiboBio (Guangzhou, China). The primary antibodies (p53, p21, cyclin D1, CDK4/6 and caspase 3 and 9) were purchased from Cell Signaling Technology (Danvers, MA, USA). The 5-pair-primers were designed by BioTNT (Shanghai, China). The Hoechst 33258 Staining kit was obtained from the Beyotime Institute of Biotechnology (Shanghai, China).

Cell culture and transfection. $\mathrm{PC1} 2$ cells were cultured in $60 \mathrm{~cm}^{2}$ dishes in an incubator at $37^{\circ} \mathrm{C}$, with $5 \% \mathrm{CO}_{2}$ and $90 \%$ humidity. The culture medium for PC12 cells consisted of $5 \%$ fetal bovine serum (FBS; Thermo Fisher Scientific, Inc., Waltham, MA, USA), $10 \%$ horse serum (Thermo Fisher Scientific) and $1 \%$ penicillin-streptomycin. PC12 cells were subcultured or the culture medium was replaced every three days.

The day before transfection, the cells were treated with trypsin and seeded into new 6-well plates at a density of 50-60\% and then cultured without antibiotics. All dsRNAs were transfected at a final concentration of $50 \mathrm{nmol} / \mathrm{l}$ with Lipofectamine RNAiMax (Thermo Fisher Scientific) according to the manufacturer's instructions. Additionally, dsRNA was replaced with Opti-MEM (Thermo Fisher Scientific), a medium in which liposomes are combined with dsRNA, for mock transfection.

Protein extraction and western blot analysis. Cells transfected with or without dsP53-285 were harvested, washed twice with phosphate-buffered saline (PBS), and then lysed in ice-cold lysis buffer containing $1 \%$ phenylmethylsulfonyl fluoride (PMSF) and $10 \%$ cocktail for $30 \mathrm{~min}$ at $4^{\circ} \mathrm{C}$. Afterwards cell lysates were centrifuged at $12,000 \mathrm{rpm}$ for $30 \mathrm{~min}$ and then loading buffer was added before storage at $-20^{\circ} \mathrm{C}$. The proteins were separated by SDS-PAGE ( $8 \%$ gels) and transferred to polyvinylidene fluoride (PVDF) membranes (Millipore, Billerica, MA, USA). BSA (5\%) was used to block nonspecific binding for $1 \mathrm{~h}$ at normal atmospheric temperature. Membranes were subsequently incubated overnight at $4^{\circ} \mathrm{C}$ with primary antibodies $(1: 1,000)$ and $\beta$-actin $(1: 2,000)$. Thereafter, the membranes were washed three times with PBS-T and then incubated with corresponding secondary antibodies at room temperature for $40 \mathrm{~min}$. Following washing three times, the protein bands were detected using the enhanced chemiluminescence method with Image Lab software (Bio-Rad Laboratories, Inc., Hercules, CA, USA) with automatic or manual exposure.

RNA extraction and real-time quantitative polymerase chain reaction ( $q P C R)$. TRIzol reagent (Thermo Fisher Scientific) was used to extract RNA from the cells. RNA (500 ng) was reverse transcribed using a reverse transcription kit (Takara Biotechnology, Dalian, China) according to the manufacturer's instructions. Quantitative PCR was performed on a 7500 Real-Time PCR system (Applied Biosystems, Foster City, CA, USA) with SYBR Premix Ex Taq II (Takara Biotechnology). The program was set for an initial denaturation step of $5 \mathrm{~min}$ at $95^{\circ} \mathrm{C}$, followed by 40 cycles at $95^{\circ} \mathrm{C}$ for $15 \mathrm{sec}, 60^{\circ} \mathrm{C}$ for $30 \mathrm{sec}$, and $72^{\circ} \mathrm{C}$ for $30 \mathrm{sec}$, with a final extension at $72^{\circ} \mathrm{C}$ for 5 min. Glyceraldehyde 3-phosphate dehydrogenase (GAPDH) was used as an internal control. Relative expression of the targeted genes was calculated using the $2^{-\Delta \Delta \mathrm{Ct}}$ method.
Cell proliferation assay. At $24 \mathrm{~h}$ after transfection, the cells at a density of $3 \times 10^{3} /$ well, were reseeded and incubated in a 96-well plate in $200 \mu \mathrm{l}$ culture medium. After 24, 48 and $72 \mathrm{~h}$ Cell Counting kit 8 (CCK8; YeaSen, Shanghai, China) was added to each well according to the manufacturer's protocol, and incubated for 0.5-4 h before absorbance measurements were performed at $450 \mathrm{~nm}$ with Gen5 software (BioTek, Winooski, VT, USA). The percentage of viable cells at every time-point was calculated in relation to the mock group. The formula for the relative cell number is: relative number $=$ absorbance of every group/absorbance of mock group.

Colony formation assay. At $24 \mathrm{~h}$ after transfection, the cells at a density of $1 \times 10^{3} /$ well were reseeded into sterile 6 -well plates with complete media for 10 days. Each medium was replaced every 3 days to maintain cell growth. The colonies were fixed with methanol for $15 \mathrm{~min}$ and then stained with crystal violet (Sigma-Aldrich, St. Louis, MO, USA) for $15 \mathrm{~min}$ at room temperature. Following washing three times with PBS, the cells were imaged with a digital camera.

Cell cycle analysis. The cells were treated with trypsin and then resuspended in $300 \mu \mathrm{l}$ of PBS. Subsequently, the cells were fixed with $700 \mu \mathrm{l}$ absolute ethyl alcohol overnight at $4{ }^{\circ} \mathrm{C}$. Cellular DNA was stained with propidium iodide (PI; $0.05 \mathrm{mg} / \mathrm{ml}$ ) and analyzed using flow cytometry (BD Biosciences, Franklin Lakes, NJ, USA).

Apoptosis assay. Cellular apoptosis was observed using the Hoechst 33258 nuclear staining kit. Briefly, after transfection, the cells were stained with Hoechst 33258 for 15 min and then washed twice with PBS before images were captured with a fluorescence microscope (Olympus, Tokyo, Japan).

Apoptosis was also objectively quantified by flow cytometry through double staining with Annexin V and PI (BD Biosciences). Briefly, at the end of every time-point, the cells were harvested and centrifuged at 1,000 rpm for $5 \mathrm{~min}$ and resuspended with binding buffer before staining with Annexin V-FITC for $10 \mathrm{~min}$ and PI for $5 \mathrm{~min}$ before flow cytometric analysis to detect apoptosis.

Mouse xenograft model. Equivalent amounts of PC12 cells $\left(\sim 20 \times 10^{6}, 200 \mu 1\right)$ infected with Lenti-dsP53-285 or Lenti-dsControl (Bio-Link, Shanghai, China) were injected subcutaneously into the left hind flanks of female athymicnude 4-week-old mice (Shanghai Institute of Materia Medica, Chinese Academy of Sciences). After one week of macroscopic tumor formation, the tumor length and width were assessed every 3 days for 21 days using calipers. Tumor volume (V) was calculated using the formula: $\mathrm{V}=$ length $\mathrm{x}$ width ${ }^{2} \mathrm{x} 0.5236 . \mathrm{On}$ the 28th day after injection, the mice were sacrificed and the tumors excised, weighed and imaged with a digital camera.

The Research Medical Ethics Committee of Ruijin Hospital Affiliated to the Medical School of Shanghai Jiaotong University, approved the present study. All applicable international, national and/or institutional guidelines for the care and use of animals were followed.

Immunohistochemistry (IHC). After fixing in $10 \%$ neutral formalin and dehydrating in concentration gradient alcohol, 
samples were embedded in paraffin, and then cut into 3- $\mu \mathrm{m}$ serial sections, which were dewaxed twice in xylene, rehydrated in alcohol and then rinsed with PBS, followed by treatment with $3 \% \mathrm{H}_{2} \mathrm{O}_{2}$ for 10 min to inactivate endogenous peroxidase. After antigen retrieval, the slides were incubated with $10 \%$ goat serum at a constant temperature to block nonspecific reactions for $10 \mathrm{~min}$. Subsequently, the sections were treated with p53 antibody (1:100 dilution) for $12 \mathrm{~h}$ at $4^{\circ} \mathrm{C}$. After being washed with PBS three times, the slides were incubated with secondary antibody at $37^{\circ} \mathrm{C}$ for $30 \mathrm{~min}$. Subsequently, they were washed again with PBS and developed in diaminobenzidine (DAB) substrate. The slides were counterstained in hematoxylin and dehydrated with ethanol and xylene before being mounted. Yellowish-brown granules at a percentage of $10 \%$ or more located in the nucleus of p53 was considered as positive.

Statistical analysis. All data are presented as the means \pm standard deviation. Statistical analyses were performed with SPSS 14.0 software (IBM SPSS, Armonk, NY, USA) and the corresponding bar graph or line chart were drawn using GraphPad Prism 7 software. Differences in measurement and enumeration data were compared using Student's t-test. A $\mathrm{P}$-value $<0.05$ was considered to indicate a statistically significant difference.

\section{Results}

dsP53-285 activates wild-type TP53 expression by targeting its promoter. A previous study has revealed that dsP53-285 can activate p53 expression by targeting its promoter sequence at the -285/-267 site (Fig. 1A) in the chimpanzee and African green monkey, which have relatively conserved sequences with human (12). Based on this evidence, we transfected synthetic dsP53-285 into PC12 cells and analyzed p53 expression. Compared to the mock and negative control (NC) groups, dsP53-285 significantly upregulated p53 mRNA and protein expression (Fig. 1B and C). Similarly, upregulation of p21 mRNA and protein were synchronous with the increased p53 expression. Since p21 expression is only induced by wild-type p53, these results directly or indirectly demonstrated that dsP53-285 induced wild-type p53 expression in PC12 cells.

dsP53-285 decreases cell viability. To evaluate the effect of enhanced p53 levels by dsP53-285, CCK8 proliferation assay was performed to assess cell viability. The results revealed a significant progressive decline in cell viability in the dsP53-285-transfected cell group compared to the mock and NC groups (Fig. 1D). The relative cell percentage after $72 \mathrm{~h}$ of transfection were $44.88 \pm 3.09(\mathrm{P}<0.001), 96.67 \pm 9.07$ and $87.93 \pm 5.56 \%(\mathrm{P}<0.01)$ in the dsP53-285, mock and $\mathrm{NC}$ groups, respectively.

dsP53-285 inhibits cell proliferation and induces cell cycle arrest and apoptosis. Colony formation assay was conducted to assess cell proliferation in the different experimental groups. As shown in Fig. 2A, the number of colony formation in the dsP53-285 group was much less than in the other two groups. Further analysis of the cell cycle progression
A

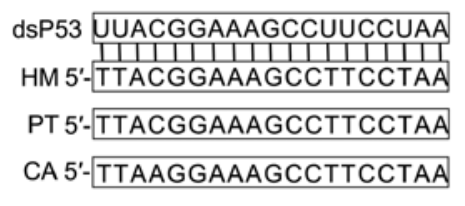

Targeted promoter sequence

$B$

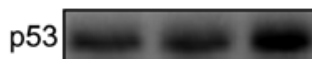

p2
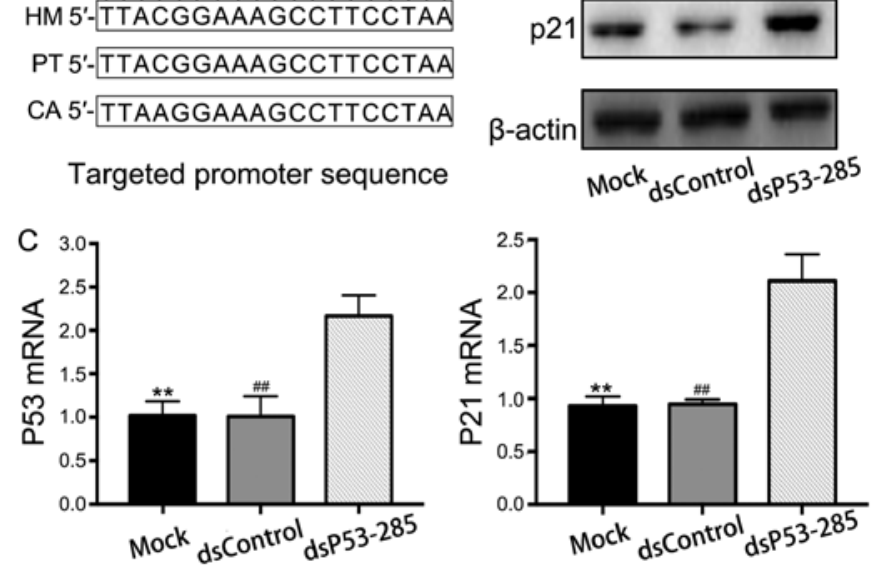

D

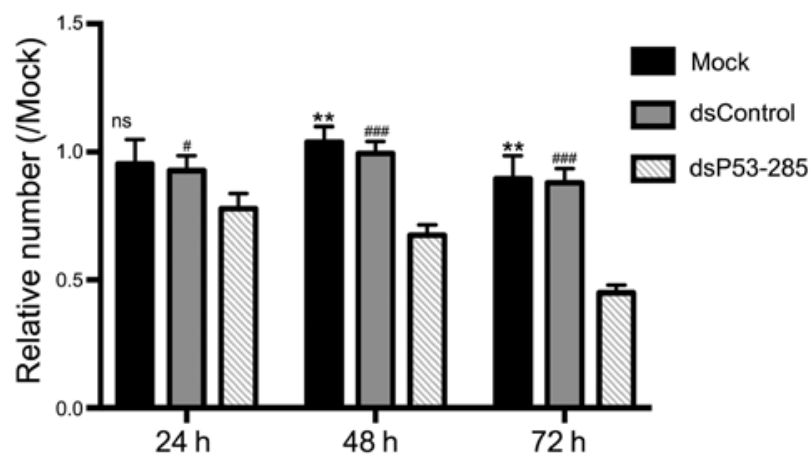

Figure 1. (A) dsP53-285 (dsP53) sequence and target alignments in human (HM), chimpanzee (PT) and African green monkey (CA). (B and C) Protein and mRNA expression levels of p53 and $\mathrm{p} 21 .{ }^{* *} \mathrm{P}<0.01$ compared to the mock group; ${ }^{\# \#} \mathrm{P}<0.01$ compared to the dsControl group. (D) Cell number relative to the mock group. ns, no statistical significance; ${ }^{* *} \mathrm{P}<0.01$ compared to the mock group; ${ }^{\#} \mathrm{P}<0.05$ and ${ }^{\# \# \#} \mathrm{P}<0.001$ compared to the dsControl group.

indicated that the dsP53-285 group was primarily arrested in the G0/G1 phase (Fig. 2B and C). The number of cells in the G0/G1 phase accounted for $56.47 \pm 4.14 \%$ in the dsP35-285 group compared to $47.23 \pm 1.29$ and $46.64 \pm 3.25 \%$ in the mock $(\mathrm{P}<0.05)$ and $\mathrm{NC}$ groups $(\mathrm{P}<0.05)$, respectively. Consequently, expression of proteins and genes related to the cell cycle, including cyclin D1 and CDK4/6 were analyzed by western blotting and qPCR. Compared to the other two groups, the levels of cyclin D1 and CDK4/6 were decreased in the dsP53-285 group (Fig. 2D and E).

Twenty-four hours after transfection, apoptotic morphologic characteristics such as cell shrinkage, rounding and decreased synaptic structures were observed in the dsP53-285 group (Fig. 3A). Subsequently, Hoechst 33258 nuclear staining kit was used to evaluate the presence of apoptosis. Compared to the other two groups, the nuclei of dsP53-285-transfected cells were dyed sapphirine-color, indicative of apoptosis (Fig. 3B). In addition, flow cytometry was used to objectively quantify apoptosis through double staining with Annexin V and PI. As illustrated in Fig. 3C and D, we concluded that dsP53-285 markedly induced progressive cellular apoptosis. At $72 \mathrm{~h}$, the apoptotic rate in the dsP53-285 group was 5.48 \pm 0.53 compared to $1.16 \pm 0.30$ and $0.94 \pm 0.48 \%$ in the mock and $\mathrm{NC}$ groups, 
A

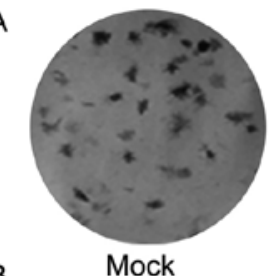

B

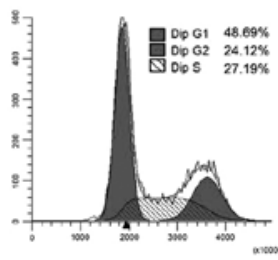

Mock

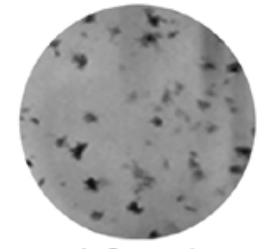

dsControl

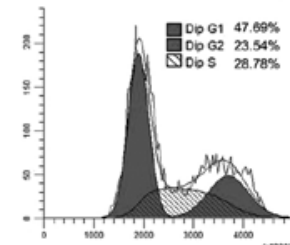

dsControl

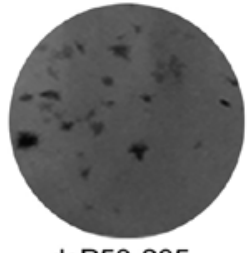

dsP53-285

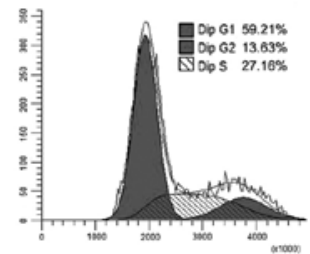

dsP53-285
D

CDK4

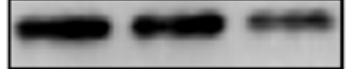

CDK6

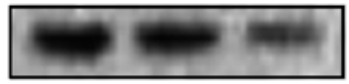

Cyclin D1

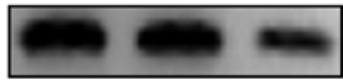

$\beta$-actin

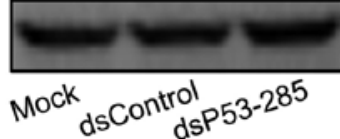

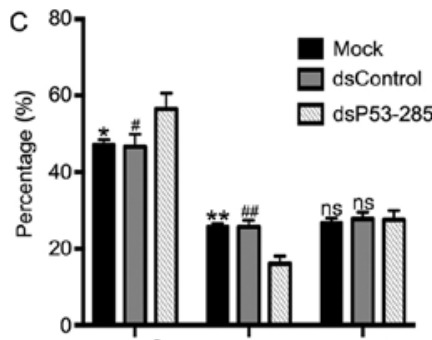

G1 phase $S$ phase $G 2$ phase
E

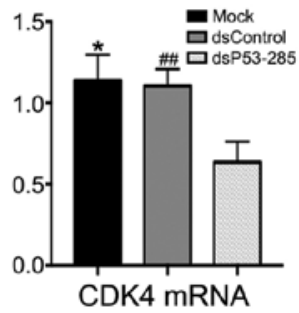

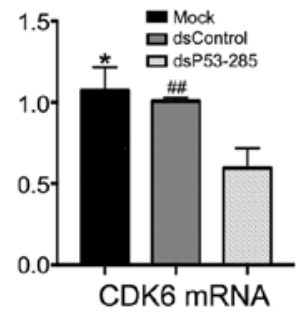

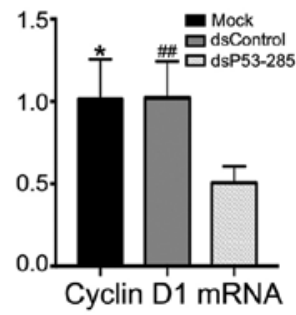

Figure 2. (A) The results of the colony formation assay. (B and C) Cell cycle arrest in the G0/G1 phase. ns, no statistical significance; ${ }^{*} \mathrm{P}<0.05$ and ${ }^{* *} \mathrm{P}<0.01$ compared to the mock group; ${ }^{~} \mathrm{P}<0.05$ and ${ }^{\# \#} \mathrm{P}<0.01$ compared to the dsControl group. (D and $\mathrm{E}$ ) Protein and mRNA expression levels of CDK4, CDK6 and cyclin $\mathrm{D} 1 .{ }^{*} \mathrm{P}<0.05$ compared to the mock group; ${ }^{\# \#} \mathrm{P}<0.01$ compared to the dsControl group.

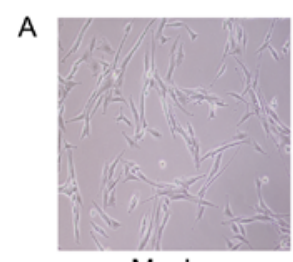

Mock

B

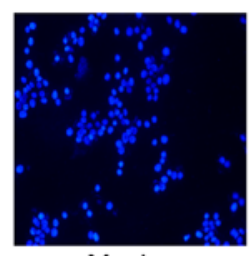

Mock
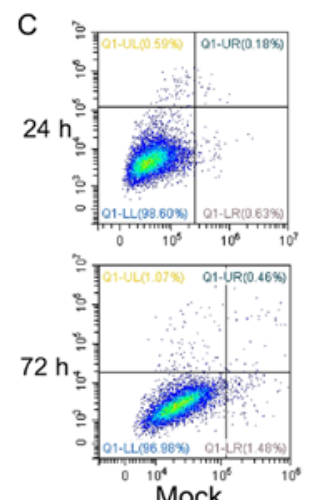

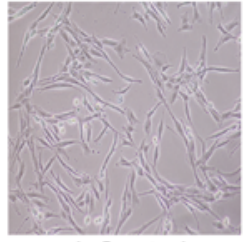

dsControl

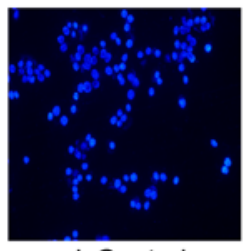

dsControl
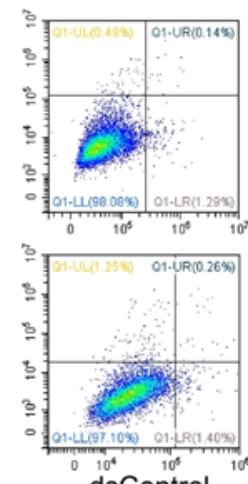

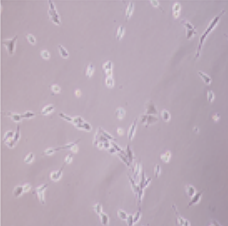

dsP53-285

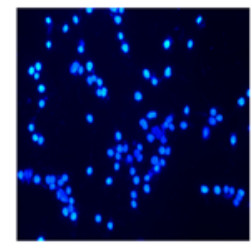

dsP53-285
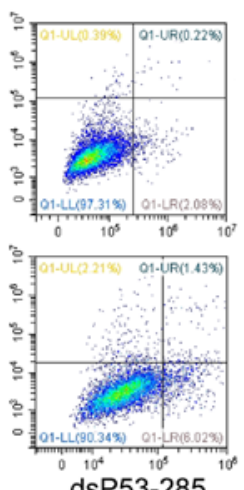
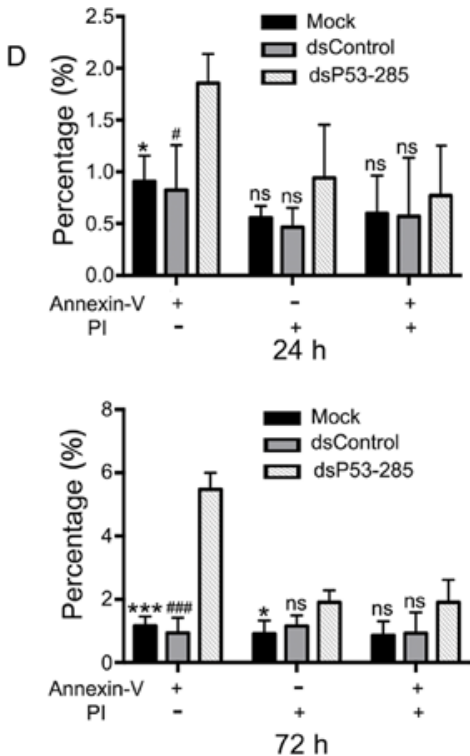

E

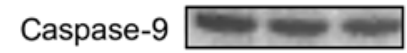

C-caspase-9 $\square$

Caspase-3 $\longrightarrow$

C-caspase-3 W

$\beta$-actin

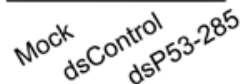

Figure 3. (A) The morphological characteristics of apoptosis under light microscopy. (B) The apoptotic induction detected by Hoechst 33258 nuclear stain assay. (C and D) dsP53-285-induced early apoptosis as detected by flow cytometry. ns, no statistical significance; ${ }^{*}<0.05$ and ${ }^{* * *} \mathrm{P}<0.001$ compared to the mock group; ${ }^{\#} \mathrm{P}<0.05$ and ${ }^{\# \# \#} \mathrm{P}<0.001$ compared to the dsControl group. (E) The expression level of (cleaved)-caspase 9 and 3. 
A
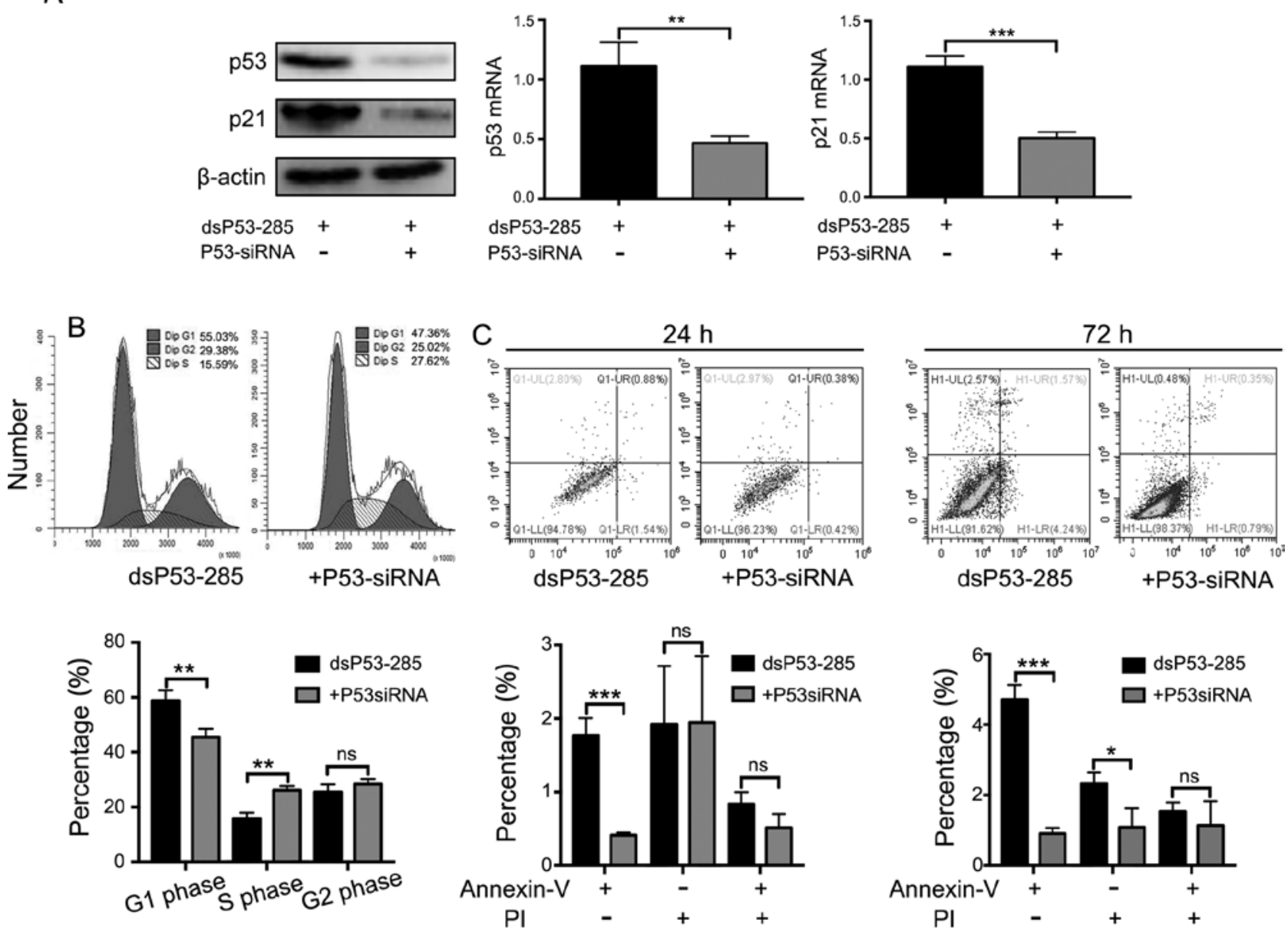

D
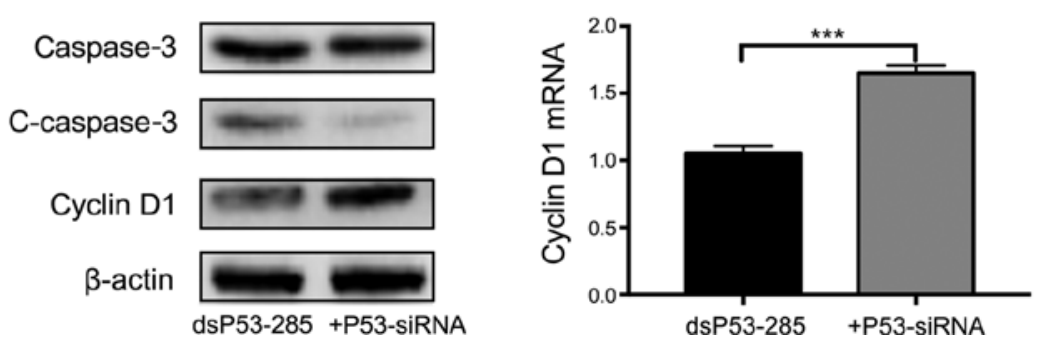

Figure 4. dsP53-285 is transfected into PC12 cells with or without siP53. (A) The decreased expression levels of p53 and p21 at both the protein and mRNA level after co-transfection with siP53. (B) Cell cycle arrest reversion after co-transfection with siP53. ns, no statistical significance; ${ }^{* * *} \mathrm{P}<0.01$. (C) Decreased number of early apoptotic cells after co-transfection with siP53. ns, no statistical significance; $\mathrm{P}<0.05 ;{ }^{* * * *} \mathrm{P}<0.001$. (D) Expression level of (cleaved)-caspase 3 and cyclin D1.

respectively. Furthermore, western blot analysis revealed that apoptosis-related proteins, caspase 9 and its downstream caspase 3, were cleaved and activated in the dsP53-285 group (Fig. 3E).

Cell cycle arrest and apoptosis induced by dsP53-285 depend on enhanced wild-type p53 expression. To determine whether the above-mentioned findings depended on the overexpression of wild-type TP53, we silenced its expression using a small interfering RNA (siP53) (Fig. 4A). Consequently, the cell cycle arrest was relieved in cells co-transfected with dsP53-285 and siP53 but not in those co-transfected with dsP53-285 and NC (Fig. 4B). The number of cells in the G0/G1 phase at $72 \mathrm{~h}$ of p53 knockdown accounted for $45.47 \pm 2.99 \%$ compared to
$58.74 \pm 3.81 \%$ in the group co-transfected with dsP53-285 and NC $(\mathrm{P}<0.01)$. Similarly, dsP53-285-induced apoptotic ability was reversed after being transfected with siP53 (Fig. 4C). Early apoptotic rate at $72 \mathrm{~h}$ of p53 knockdown was $0.91 \pm 0.16$ vs. $4.71 \pm 0.41 \%$ in the other group. Consistent with the results of flow cytometry, the expression level of cyclin D1 was upregulated and cleaved-caspase 3 was downregulated after transfection with siP53 (Fig. 4D). These data revealed that cell cycle arrest and apoptosis induced by dsP53-285 completely depended on the enhanced wild-type p53.

dsP53-285 reduces xenograft tumor grow in vivo. Through the construction of a recombinant lentivirus expression vector, PC12 cells stably expressing p53 were transplanted into nude 
A

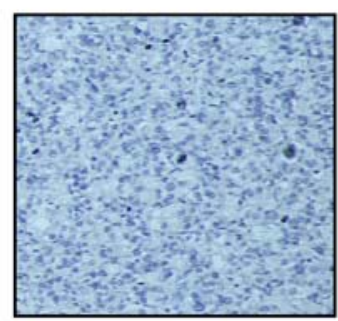

dsControl

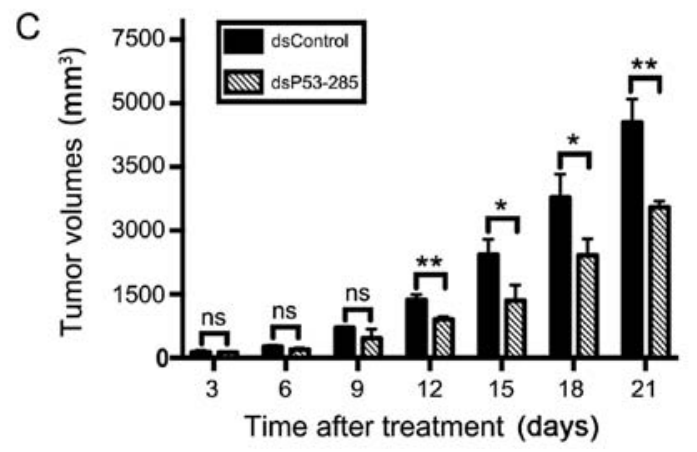

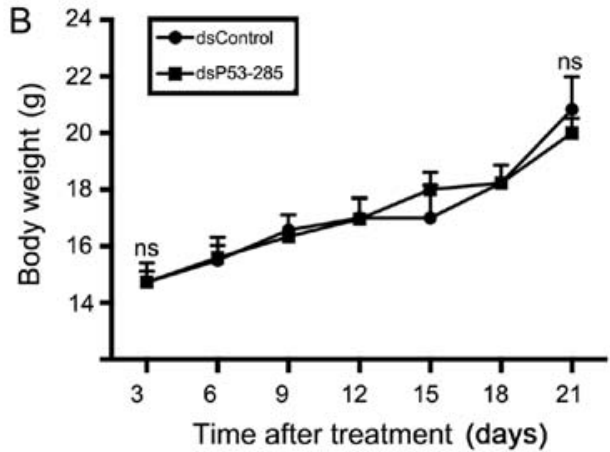

D

dsControl

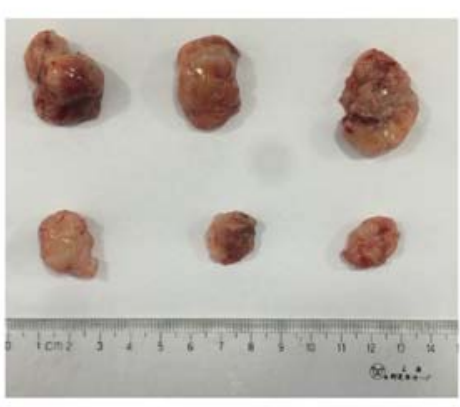

Figure 5. In vivo experiments. (A) Expression of p53 in samples from transplanted tumors (magnification, x200). (B) Mouse body weight. ns, no statistical significance. (C) Tumor volumes. ns, no statistical significance; ${ }^{*} \mathrm{P}<0.05 ;{ }^{* *} \mathrm{P}<0.01$. (D) Photographs of isolated xenograft tumors.

mice, referred to as dsP53-285 group. At the end of the experiments, the tumors were isolated, weighed and subjected to immunohistochemical staining. Predictably, positive expression of p53 was observed in the dsP53-285 group rather than in the control group transfected with Lenti-dsControl vector cells (Fig. 5A). Compared to the control group, the mean tumor volume in the dsP53-285 group was significantly smaller $\left(3,548 \pm 148\right.$ vs. $5,547 \pm 555 \mathrm{~mm}^{3}, \mathrm{P}<0.01$; Fig. $5 \mathrm{C}$ and D), but without significant difference in mouse body weight (22.00 \pm 0.53 vs. $22.83 \pm 1.15$ g, $\mathrm{P}>0.05$; Fig. $5 \mathrm{~B})$.

\section{Discussion}

TP53 is an established tumor suppressor gene with frequent inactivating mutations in various human types of cancer, including PHEO (13-16). As a dsRNA targeted to TP53 promoter sequence, dsP53-285 has been ascertained to activate the wild-type TP53 expression with antitumor effects in human cancers $(17,18)$.

Currently, there are no clinically reliable markers for the early diagnosis of malignant PHEO, with the exception of the gold standard of finding ectopic chromaffin tissue. However, patients with metastatic disease miss optimal treatment opportunities and only partially benefit from conventional chemotherapy. However, little is known about whether dsP53-285 has any anti-PHEO effects through the activation of wild-type TP53.

The data presented in the present study demonstrated that synthetic dsP53-285 could induce robust wild-type TP53 expression. As a downstream target of wild-type but not mutant TP53, p21 expression at both the mRNA and protein level was upregulated by dsP53-285, indirectly revealing that dsP53-285 could induce p21 expression. Preliminary proliferation assay results demonstrated that dsP53-285 directly inhibited progressive PC12 cell viability. Moreover, we ascertained that transfection of dsP53-285 inhibited tumor cell viability through the induction of G0/G1-phase arrest and apoptosis, both of which were consistent with the altered expression level of related mRNAs and proteins. In addition, dsP53-285 impeded tumor growth in vivo. Notably, dsP53-285-induced antitumor effects were reversed following co-transfection with siP53. This finding further indicated that dsP53-285 enhanced p53 expression and functions.

Dysregulation of the cell cycle that causes abnormal cell proliferation to promote tumor progression is a universal phenomenon of tumors. The cell cycle is regulated by cyclin-CDK complexes, such as cyclin D1-CDK4/6 complexes, which are required for the transition from the G0/G1 phase to the $\mathrm{S}$ phase. Besides, p21 protein mediates p53-dependent cell cycle arrest by binding and inhibiting cyclin-CDK complexes (19-23). Other researchers as well as we have demonstrated that dsP53-285 induces G0/G1 phase arrest and supresses the expression of cyclin D1 and CDK4/6 $(17,18)$. Following p53 knockdown, the cell cycle arrest induced by dsP53-285 was reversed, revealing that dsP53-285 activated wild-type p53 and p21 to induce G0/G1 phase arrest.

Apoptosis contributes to maintaining homeostasis of organismal internal environment through the orderly and effective clearance of damaged cells. Thus, its dysregulation is frequently associated with excessive proliferation that eventually leads to tumor formation and drug resistance $(24,25)$. Research reveals that p53 regulates the BCL-2 family of proteins regarded as the most vital regulators of apoptosis (26). In the present study, we firstly demonstrated that dsP53-285 could induce apoptosis through the upregulation of cleaved-caspase 9 and 3, an activated form of caspase proteins. 
However, we did not detect expression changes in the levels of BCL-2 family of proteins such as BCL-2 and Bax.

RNAa involves specific and nonspecific target genes. Additionally, it has a prolonged duration that could last up to two weeks $(27,28)$. In regard to oncotherapy, RNAa has primarily been focused on activating mutant anti-oncogenes that are universally inactivated in human tumors, irrespective of mutant oncogenes. Thus, RNAa appears to be an effective, specific and widespread strategy for cancer therapy.

In conclusion, our results strongly suggest that dsP53-285 could inhibit proliferation and induction of apoptosis in PC12 cells through upregulation of wild-type TP53. Our findings highlight the therapeutic potential of dsP53-285 for PHEO.

\section{Acknowledgements}

The National Natural Science Foundation of China (nos. 81272936 and 81602215) and the Shanghai Nature Science Foundation (no. 17ZR1417300) funded this study. We would also like to sincerely thank professor Jianqing Ding (Department of Neurology, Ruijin Hospital Affiliated to Medical School of Shanghai Jiaotong University) for his guidance with the experiments and the provision of laboratory place.

\section{References}

1. Walther MM, Herring J, Enquist E, Keiser HR and Linehan WM von Recklinghausen's disease and pheochromocytomas. J Urol 162: 1582-1586, 1999.

2. Edström Elder E, Hjelm Skog AL, Höög A and Hamberger B: The management of benign and malignant pheochromocytoma and abdominal paraganglioma. Eur J Surg Oncol 29: 278-283, 2003.

3. Schürmeyer T, Dralle H, Schuppert F and von zur Mühlen A: Preoperative diagnosis of suspected pheochromocytoma - retrospective assessment of diagnostic criteria. Acta Med Austriaca 15: 106-108, 1988 (In German).

4. Roman-Gonzalez A and Jimenez C: Malignant pheochromocytoma-paraganglioma: Pathogenesis, TNM staging, and current clinical trials. Curr Opin Endocrinol Diabetes Obes 24: 174-183, 2017.

5. Kopf D, Goretzki PE and Lehnert H: Clinical management of malignant adrenal tumors. J Cancer Res Clin Oncol 127: $143-155,2001$.

6. Muller PA and Vousden KH: Mutant p53 in cancer: New functions and therapeutic opportunities. Cancer Cell 25: 304-317, 2014.

7. Vousden KH and Lu X: Live or let die: The cell's response to p53. Nat Rev Cancer 2: 594-604, 2002.

8. Oren M: Decision making by p53: Life, death and cancer. Cell Death Differ 10: 431-442, 2003.

9. Vousden KH and Lane DP: p53 in health and disease. Nat Rev Mol Cell Biol 8: 275-283, 2007.
10. Lin SR, Lee YJ and Tsai JH: Mutations of the p53 gene in human functional adrenal neoplasms. J Clin Endocrinol Metab 78: 483-491, 1994.

11. Yoshimoto T, Naruse M, Zeng Z, Nishikawa T, Kasajima T, Toma H, Yamamori S, Matsumoto H, Tanabe A, Naruse K, et al: The relatively high frequency of p53 gene mutations in multiple and malignant phaeochromocytomas. J Endocrinol 159: 247-255, 1998.

12. Huang V, Qin Y, Wang J, Wang X, Place RF, Lin G, Lue TF and Li LC: RNAa is conserved in mammalian cells. PLoS One 5: e8848, 2010.

13. Hollstein M, Sidransky D, Vogelstein B and Harris CC: p53 mutations in human cancers. Science 253: 49-53, 1991.

14. Milner J, Medcalf EA and Cook AC: Tumor suppressor p53: Analysis of wild-type and mutant p53 complexes. Mol Cell Biol 11: 12-19, 1991.

15. Kato S, Han SY, Liu W, Otsuka K, Shibata H, Kanamaru R and Ishioka $C$ : Understanding the function-structure and function-mutation relationships of $\mathrm{p} 53$ tumor suppressor protein by high-resolution missense mutation analysis. Proc Natl Acad Sci USA 100: 8424-8429, 2003.

16. Schlomm T, Iwers L, Kirstein P, Jessen B, Köllermann J, Minner S, Passow-Drolet A, Mirlacher M, Milde-Langosch K, Graefen M, et al: Clinical significance of p53 alterations in surgically treated prostate cancers. Mod Pathol 21: 1371-1378, 2008.

17. Ge Q, Wang C, Ruan Y, Chen Z, Liu J and Ye Z: Overexpression of p53 activated by small activating RNA suppresses the growth of human prostate cancer cells. Onco Targets Ther 9: 231-241, 2016.

18. Wang C, Ge Q, Zhang Q, Chen Z, Hu J, Li F and Ye Z: Targeted p53 activation by saRNA suppresses human bladder cancer cells growth and metastasis. J Exp Clin Cancer Res 35: 53, 2016.

19. Brugarolas J, Chandrasekaran C, Gordon JI, Beach D, Jacks T and Hannon GJ: Radiation-induced cell cycle arrest compromised by p21 deficiency. Nature 377: 552-557, 1995.

20. Waldman T, Kinzler KW and Vogelstein B: p21 is necessary for the p53-mediated G1 arrest in human cancer cells. Cancer Res 55: 5187-5190, 1995.

21. Del Sal G, Murphy M, Ruaro E, Lazarevic D, Levine AJ and Schneider C: Cyclin D1 and p21/waf1 are both involved in p53 growth suppression. Oncogene 12: 177-185, 1996.

22. Xiong Y, Hannon GJ, Zhang H, Casso D, Kobayashi R and Beach D: p21 is a universal inhibitor of cyclin kinases. Nature 366: 701-704, 1993.

23. Waga S, Hannon GJ, Beach D and Stillman B: The p21 inhibitor of cyclin-dependent kinases controls DNA replication by interaction with PCNA. Nature 369: 574-578, 1994.

24. Plati J, Bucur O and Khosravi-Far R: Dysregulation of apoptotic signaling in cancer: Molecular mechanisms and therapeutic opportunities. J Cell Biochem 104: 1124-1149, 2008.

25. Fulda S: Tumor resistance to apoptosis. Int J Cancer 124: 511-515, 2009.

26. Groeger AM, Esposito V, De Luca A, Cassandro R, Tonini G, Ambrogi V, Baldi F, Goldfarb R, Mineo TC, Baldi A, et al: Prognostic value of immunohistochemical expression of $\mathrm{p} 53$, bax, Bcl-2 and Bcl-xL in resected non-small-cell lung cancers. Histopathology 44: 54-63, 2004.

27. Li LC, Okino ST, Zhao H, Pookot D, Place RF, Urakami S, Enokida H and Dahiya R: Small dsRNAs induce transcriptional activation in human cells. Proc Natl Acad Sci USA 103: 17337-17342, 2006.

28. Place RF, Noonan EJ, Földes-Papp Z and Li LC: Defining features and exploring chemical modifications to manipulate RNAa activity. Curr Pharm Biotechnol 11: 518-526, 2010. 